\title{
The LUT-SR Family of Uniform Random Number Generators for FPGA Architectures
}

\author{
David B. Thomas, Member, IEEE, and Wayne Luk, Fellow, IEEE
}

\begin{abstract}
Field-programmable gate array (FPGA) optimized random number generators (RNGs) are more resource-efficient than software-optimized RNGs because they can take advantage of bitwise operations and FPGA-specific features. However, it is difficult to concisely describe FPGA-optimized RNGs, so they are not commonly used in real-world designs. This paper describes a type of FPGA RNG called a LUT-SR RNG, which takes advantage of bitwise XOR operations and the ability to turn lookup tables (LUTs) into shift registers of varying lengths. This provides a good resource-quality balance compared to previous FPGA-optimized generators, between the previous high-resource high-period LUT-FIFO RNGs and low-resource low-quality LUTOPT RNGs, with quality comparable to the best software generators. The LUT-SR generators can also be expressed using a simple $\mathrm{C}++$ algorithm contained within this paper, allowing 60 fully-specified LUT-SR RNGs with different characteristics to be embedded in this paper, backed up by an online set of very high speed integrated circuit hardware description language (VHDL) generators and test benches.
\end{abstract}

Index Terms-Equidistribution, field-programmable gate array (FPGA), uniform random number generator (RNG).

\section{INTRODUCTION}

$\mathbf{M}^{\circ}$ ONTE CARLO applications are ideally suited to fieldprogrammable gate arrays (FPGAs) because of the highly parallel nature of the applications, and because it is possible to take advantage of hardware features to create very efficient random number generators (RNGs). In particular, uniform random bits are extremely cheap to generate in an FPGA, as large numbers of bits can be generated per cycle at high clock rates using lookup tables [1], or first-in-firstout (FIFO) queues [2]. In addition, these generators can be customized to meet the exact requirements of the application, both in terms of the number of bits required per cycle, and for the FPGA architecture of the target platform.

Despite these advantages, FPGA-optimized generators are not widely used in practice, as the process of constructing a generator for a given parameterization is time consuming, in terms of both developer man hours and CPU time. While it is possible to construct all possible generators ahead of time, the resulting set of cores would require many megabytes,

Manuscript received January 24, 2012; accepted March 21, 2012. Date of publication April 26, 2012; date of current version March 18, 2013. This work was supported in part by the U.K. Engineering and Physical Sciences Research Council under Grant EP/D062322/1 and Grant EP/C549481/1.

D. B. Thomas is with the Department of Electrical and Electronic Engineering, Imperial College London, London SW7, U.K. (e-mail: dt10@imperial.ac.uk).

W. Luk is with the Department of Computing, Imperial College London, London SW7, U.K. (e-mail: wl@ doc.ic.ac.uk).

Digital Object Identifier 10.1109/TVLSI.2012.2194171 and be difficult to integrate into existing tools and design flows. Faced with these unpalatable choices, engineers under time constraints understandably choose less efficient methods, such as combined Tausworthe generators [3] or parallel linear feedback shift registers (LFSRs).

This paper describes a family of generators which makes it easier to use FPGA-optimized generators by providing a simple method for engineers to instantiate an RNG that meets the specific needs of their application. Specifically, it shows how to create a family of generators called LUT-SR RNGs, which use LUTs as shift registers to achieve high quality and long periods, while requiring very few resources. The main contributions of this paper are as follows:

1) a type of FPGA-optimized uniform RNG called a LUT-SR generator is presented which uses LUT-based shift registers to implement generators with periods of $2^{1024}-1$ or more, using two LUTs and two flip flops (FFs) per generated random bit;

2) an algorithm for describing LUT-SR RNGs using five integers is given, along with a set of open-source test benchs and tools;

3) tables of 60 LUT-SR RNGs are presented, covering output widths from 32 up to 624 , with periods from $2^{1024}-1$ up to $2^{19937}-1$;

4) a theoretical quality analysis of the given RNGs in terms of equidistribution and a comparison with other software and hardware RNGs are carried out.

The LUT-SR family was first presented in a conference paper [4], which concentrated on the practical aspects of constructing and using these generators. This paper adds Section $\mathrm{V}$, which describes the method used to find maximum period generators, Section VI, which describes the process used to select the highest quality generators, and Section VIII, which gives a rigorous theoretical quality analysis in terms of equidistribution.

\section{OVERVIEW OF BINARY LINEAR RNGS}

The LUT-SR RNGs are part of a large family of RNGs, all of which are based on binary linear recurrences. This family includes many of the most popular contemporary software generators, such as the Mersenne Twister (MT-19937) [5], the Combined Tausworthe (TAUS-113) [3], SFMT [6], WELL [7], and TT-800 [8]. This section gives an overview of the underlying maths, and describes existing binary linear RNGs used in FPGAs. 


\section{A. Binary Linear RNGs}

Binary linear recurrences operate on bits (binary digits), where addition and multiplication of bits is implemented using exclusive-or $(\oplus)$ and bitwise-and $(\otimes) .{ }^{1}$ The recurrence of an RNG with $n$-bit state and $r$-bit outputs is defined as

$$
\begin{aligned}
\mathbf{x}_{i+1} & =\mathbf{A} \mathbf{x}_{i} \\
\mathbf{y}_{i+1} & =\mathbf{B} \mathbf{x}_{i+1}
\end{aligned}
$$

where $\mathbf{x}_{i}=\left(x_{i, 1}, x_{i, 2}, \ldots, x_{i, n}\right)^{\top}$ is the $n$-bit state of the generator, $\mathbf{y}_{i}=\left(y_{i, 1}, y_{i, 2}, \ldots, y_{i, r}\right)^{\top}$ is the $r$-bit output of the generator, $\mathbf{A}$ is an $n \times n$ binary transition matrix, and $\mathbf{B}$ is an $r \times n$ binary output matrix. Because the state is finite, and the recurrence is deterministic, eventually the state sequence $\mathbf{x}_{0}, \mathbf{x}_{1}, \mathbf{x}_{2}, \ldots$ must start to repeat.

The minimum value $p$ such that $\mathbf{x}_{i+p}=\mathbf{x}_{i}$ is called the period of the generator, and one goal in designing RNGs is to achieve the maximum period of $p=2^{n}-1$. A period of $2^{n}$ cannot be achieved because it is impossible to choose $\mathbf{A}$ such that $\mathbf{x}_{0}=\mathbf{0}$ maps to anything other than $\mathbf{x}_{1}=\mathbf{0}$. This leads to two sequences in a maximum period generator: a degenerate sequence of length 1 which contains only zero, and the main sequence which iterates through every possible nonzero $n$-bit pattern before repeating. A necessary and sufficient condition for a generator to have maximum period is that the characteristic polynomial $P(z)$ of the transition matrix A must be primitive [1].

The matrix $\mathbf{B}$ is used to transform the internal RNG state into the random output bits produced by the generator. In the simplest case, we have $r=n$ and $\mathbf{B}=\mathbf{I}$, which means the state bits are used directly as random output bits, but in many generators most of the internal state bits are not sufficiently random. In these cases, $r<n$, and either some state bits are not propagated through to the output, or multiple state bits are XOR'd together to produce each output bit.

The quality of a generator is measured in two ways: empirical tests, which look at generated sequences of numbers, and theoretical tests, which consider mathematical properties of the entire number sequence. Examples of empirical tests are to track the distribution of $1 \mathrm{~s}$ versus $0 \mathrm{~s}$, or to examine the Fourier transform of the uniform sequence, and to see if the observed behavior deviates significantly from the behavior of an ideal uniform source. Many such tests are gathered into batteries such as TestU01 [9], which examine long output sequences from a given RNG to find evidence of nonrandomness.

Theoretical randomness of binary linear generators is measured using equidistribution [3], which describes how evenly distributed the output sequence is in multiple dimensions. Equidistribution is defined in terms of a resolution $l$, which is the number of most significant bits considered from the output, and a dimension $d$, which is the number of consecutive output samples. If a generator is $(d, l)$-distributed, then every possible length $d$ sequence will be observed in the output sequence the same number of times. For example, if a 32-bit generator is $(3,16)$-distributed, then all possible 16-bit output triples will be equally likely. Alternatively, if a generator is $(d, r)$ distributed, that is, it is equidistributed to $d$ dimensions over

\footnotetext{
${ }^{1}$ This is the same as operating modulo-2, or in the Galois field GF(2).
}

the output bit width, and it is initialized to an unknown random state $x_{0}$, then observing $y_{1}, \ldots, y_{d-1}$ gives no information at all about what $y_{d}$ will be. However, observing $y_{1}, \ldots, y_{d}$ may allow us to make predictions about the value of $y_{d+1}$, or in some cases allow us to predict it precisely.

There are three stages when designing such a generator.

1) Describe a family of generators $G_{F}$, such that each member of $G_{F}$ can be efficiently implemented in the target architecture. However, only some members of $G_{F}$ will have the maximum period property.

2) Extract a maximum period subset $G_{M} \subset G_{F}$, such that all members of $G_{M}$ implement a matrix $\mathbf{A}$ with a primitive characteristic polynomial. This is achieved either by randomly selecting and testing members of $G_{F}$, or by exhaustive enumeration if $\left|G_{F}\right|$ is small.

3) Find the generator $g_{I} \in G_{M}$ which produces the output stream with highest statistical quality, either by considering multiple members with different $\mathbf{A}$ matrices, or by trying many different $\mathbf{B}$ matrices for a single transition matrix.

The selected RNG instance $g_{I} \in G_{F}$ can then be expressed as code [e.g., C or VHDL] and used in the target architecture.

\section{B. LUT-Optimized (LUT-OPT) RNGs}

LUT-OPT generators [1] are a family of generators with a matrix $A$ where each row and column contains $t-1$ or $t$ 1s. In hardware terms, this means that each row maps to a $t-1$ or $t$ input XOR gate, and so can be implemented in a single $t$ input LUT. Thus if the current vector state is held in a register, each bit of the new vector state can be calculated in a single LUT, and an $r$-bit generator can be implemented in $r$ fully utilized LUT-FFs. The basic structure of a LUT-OPT generator is shown in Fig. 1(a).

A simple example of a maximum period LUT-OPT generator with $r=6$ and $t=3$ is given by the recurrence

$$
\mathbf{A}=\left[\begin{array}{llllll}
0 & 1 & 1 & 0 & 0 & 0 \\
0 & 1 & 1 & 0 & 0 & 1 \\
0 & 1 & 0 & 1 & 0 & 0 \\
1 & 0 & 0 & 0 & 1 & 0 \\
1 & 0 & 0 & 0 & 0 & 1 \\
1 & 0 & 0 & 1 & 1 & 0
\end{array}\right], \quad\left[\begin{array}{l}
x_{i+1,1} \\
x_{i+1,2} \\
x_{i+1,3} \\
x_{i+1,4} \\
x_{i+1,5} \\
x_{i+1,6}
\end{array}\right]=\left[\begin{array}{l}
x_{i, 2} \oplus x_{i, 3} \\
x_{i, 2} \oplus x_{i, 3} \oplus x_{i, 6} \\
x_{i, 2} \oplus x_{i, 4} \\
x_{i, 1} \oplus x_{i, 5} \\
x_{i, 1} \oplus x_{i, 6} \\
x_{i, 1} \oplus x_{i, 4} \oplus x_{i, 5}
\end{array}\right] .
$$

Such matrices can be found for all $t \geq 3$ and $r \geq 4$, and are practical for generating up to $\sim 1000$ uniform bits per cycle. LUT-OPT generators have two key advantages.

1) Resource efficiency: Each additional bit requires one additional LUT and FF, so resource usage scales linearly, and generating $r$ bits per cycle requires $r$ LUT-FFs.

2) Performance: The critical path in terms of logic is a single LUT delay, so the generators are extremely fast, so usually the clock net is the limiting factor, with routing delay and congestion only becoming a factor for large $n$.

However, these advantages are countered by a number of disadvantages. 


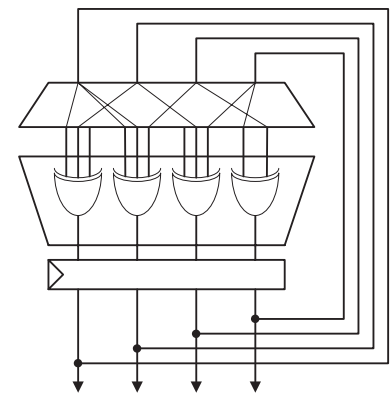

(a)

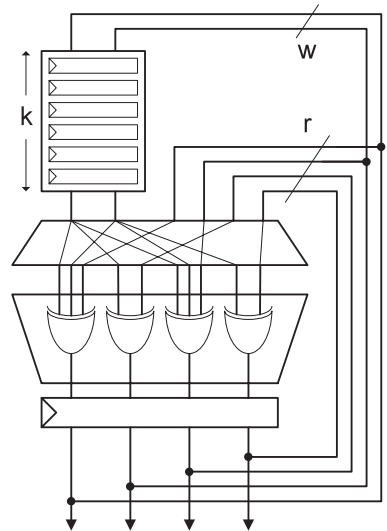

(b)

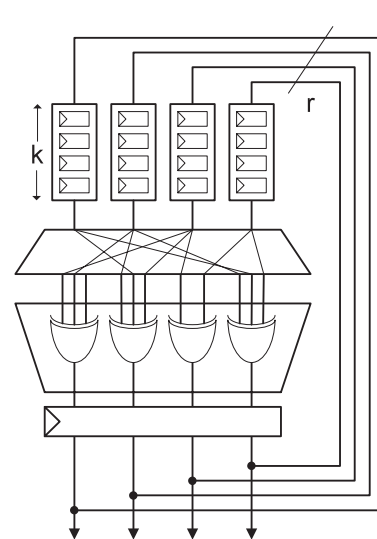

(c)

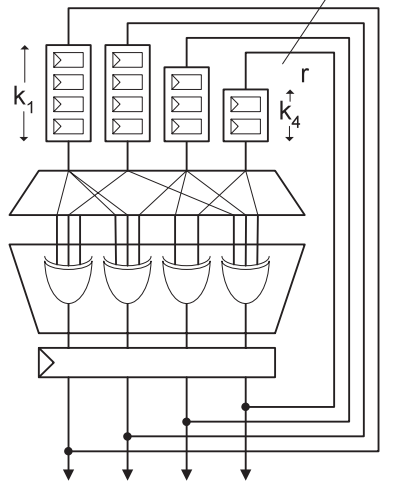

(d)

Fig. 1. Connection characteristics of four different types of FPGA-optimized binary linear RNG: (a) LUT-OPT RNG; (b) LUT-FIFO RNG; (c) FIFO of LUT-OPT RNGs; and (d) LUT-SR RNG.

1) Complexity: Each $(r, t)$ combination requires a unique matrix of connections, which must be found using specialized software. If these matrices are randomly constructed (as in previous work), then it is difficult to compactly encode these matrices, so it is difficult for FPGA engineers to make use of the RNGs.

2) Quality: The random bits are formed as a linear combination of random bits produced in the previous cyclewhen $t=3$, some of the new bits will be a simple twoinput XOR of bits from the previous cycle. The impact of this lag-1 linear dependence is minimal in modern FPGAs where $t \geq 5$, and also diminishes quickly as $r$ is increased, but remains a source of concern.

3) Period: In order to achieve a period of $2^{n}-1$, it is necessary to choose $r=n$, even if far fewer than $n$ bits are needed per cycle. An absolute minimum safe period for a hardware generator is $2^{64}-1$, but it is preferable to have much larger periods of $2^{1000}-1$ or more.

4) Seeding: It is necessary to initialize RNGs with a chosen state at run time, so that different hardware instances of the same RNG algorithm will generate different random streams. In a LUT-optimized generator, it is possible to implement serial loading of state using one LUT input per RNG bit to select between RNG and load mode, but in practice, for a randomly chosen matrix $A$, only parallel loading is possible.

\section{LUT-FIFO RNGS}

One way of removing the quality and period problems is provided by LUT-FIFO generators [2]. These augment the $r$ bits of state held in FFs with an additional depth- $k$ width- $w$ first-in-first-out (FIFO), for a total period of $2^{n}-1$, where $n=r+w k$, shown in Fig. 1(b). LUT-FIFO generators can provide long periods such as $2^{11213}-1$ and $2^{19937}-1$, but also have the following disadvantages.

1) For reasonable efficiency, the FIFO needs to be implemented using a block RAM, a relatively expensive resource which one would usually prefer to use elsewhere in a design.
2) The wordwise granularity of block-RAM-based FIFOs reduces the flexibility in the choice of $r$, as it can only be varied in multiples of $k$.

These are mild disadvantages when compared to the quality and period problems of LUT-optimized generators that have been eliminated, but LUT-FIFO generators also make the problems of complexity and efficient initialization slightly worse. If extremely high quality and period are needed, then LUT-FIFO generators present the fastest and most efficient solution, but few applications actually require such high levels of quality, particularly given the need for expensive blockRAM resources.

\section{Software RNGs}

In addition to the hardware-optimized LUT-OPT and LUTFIFO generators, a number of generators designed for software architectures have been ported to FPGA architectures.

Combined Tausworthe [3] - Software generators which use word-level shift, XOR, and AND operations to construct simple recurrences with distinct periods, which are then combined using XOR to produce a much longer period generator.

Mersenne Twister [5] - This uses the same word-level operators as the Combined Tausworthe, combined with a large RAM-based queue, to create a software generator with a fairly good equidistribution and the extremely long period of $2^{19937}-1$.

WELL [10]-This generator uses techniques similar to the Mersenne Twister, but uses a more complex recurrence step involving multiple memory accesses per sample, to achieve the maximum possible equidistribution at the same period as the Mersenne Twister.

All the software generators are designed with word-level instructions in mind, and so tend to be inefficient in terms of resources consumed per bit generated. In this paper, we show the LUT-SR generator, which sits between the LUT-optimized and LUT-FIFO generators. It fixes all problems related to complexity and serial seeding found with both generators, and provides much higher periods than LUT-OPT generators for a cost of one extra LUT-FF per bit, while eliminating the blockRAM resource needed for an LUT-FIFO RNG. 


\section{LUT-SR RNG}

Modern FPGAs allow LUTs to be configured in a number of different ways, such as basic ROMs, RAMs, and shift registers. Configuring LUTs as shift registers provides an attractive means of adding more storage bits to a binary linear generator: for example, adding one Xilinx SRL32 to a LUT-optimized $r$ bit generator allows the state size to be increased to $n=r+32$. This represents a degenerate form of an LUT-FIFO generator, with $k=32$ and $w=1$.

However, while the FIFO in a LUT-FIFO RNG is usually an expensive block RAM, LUT-based shift registers are very cheap-almost as cheap as the LUTs used to build the XOR gates. So it now becomes economical to use $r$ shift registers, one per output bit, increasing the potential state to $n=r(1+$ $k$ ). If we assume $k=32$ (as found in modern FPGAs), and a modest RNG output width of $r=32$, the state size increases to $n=1056$. This provides a potential period of $2^{1056}-1$ for a cost of 64 LUTs, as compared to a period of $2^{64}-1$ for a LUT-optimized generator with the same resource usage.

It might be tempting to simply configure all shift registers with the same length, in an attempt to maximize the period for a given number of resources, but this cannot provide a maximum period generator. Instead, it would result in $1+k$ independent $r$-bit generators, with a sample taken from each on successive cycles, shown in Fig. 1(c). In LUT-FIFO generators, this problem is avoided by making each new output bit dependent on one bit from the previous cycle, with the remaining $t-1$ or $t-2$ bits provided by the FIFO output. This lag-1 dependency is not ideal, but is generally benign as the LUT-FIFO uses deep block-RAM-based FIFOs.

Individual one bit shift registers enable a different solution that allows large periods to be achieved, while also improving the rate of mixing within the generator state. Each of the $r$ shift registers can be assigned some specific length $k_{i} \leq k$, reducing the state size to $n=\sum_{i=1}^{r}\left(1+k_{i}\right)$. One solution would be to randomly configure each shift register as $k_{i}=k$ or $k_{i}=k-1$, giving a period $r k<n<r(1+k)$. But a much more interesting solution is to randomly choose $1<k_{i} \leq k$, subject to the constraint $\exists i, j: i \neq j \wedge \operatorname{gcd}\left(k_{i}+1, k_{j}+1\right)=1$. This allows for much more rapid mixing between bits within the state, while still providing necessary (but not sufficient) conditions for mixing within the state.

Fig. 1(d) shows the new LUT-SR style of generator. All four generators shown in the figure may seem superficially similar, but actually provide quite different tradeoffs in terms of quality versus resource usage.

Both the LUT-OPT and LUT-FIFO RNGs were originally developed and presented without much consideration of how to initialize the generator state. Initialization is very important, as it is common to instantiate multiple parallel RNGs implementing the same algorithm, and each must be initialized with a distinct seed (initial state vector).

Given an $l$-input LUTs, we can implement a $t=l$ LUToptimized RNG, but there is no way to read or write the RNG state. ${ }^{2}$ Loading the state in parallel only allows a $t=l-2$ RNG, as one LUT input is needed to select when to load, and

${ }^{2}$ Excluding device-specific FF read/write chains, such as provided in Stratix.

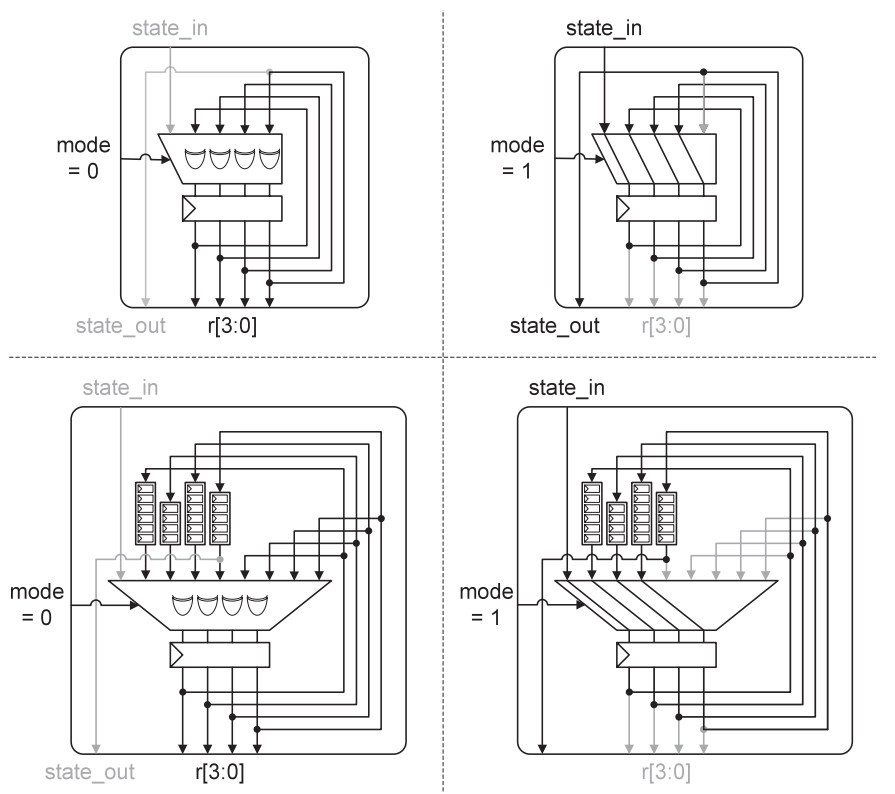

Fig. 2. Normal versus load paths for serial seeding of RNGs.

the other is needed to provide the new 1-bit value. In a fourLUT architecture, this implies $t=2$, which is not possible (some bits would be direct copies of bits from the previous cycle), so two LUTs per bit would be needed. Parallel loading also means that the RNG initialization circuitry (which is only needed at the start of simulations, and so should be as small as possible) must contain $O(r)$ resources, so even in the best case the resources per RNG have doubled.

A better approach is to find a cycle through the matrix $A$, and use this to implement a 1-bit shift register through the RNG. This only requires one bit per LUT (to select between RNG and load mode), so allows $t=l-1$, requiring only one LUT per bit in a four-LUT architecture, and allowing an efficient serial initialization circuit, no matter how large $r$ is. This approach can be extended to the LUT-FIFO RNG as well, as in any maximum period generator there must exist a cycle running through all state bits in the RNG.

However, if the matrix $A$ is randomly chosen, we must find a Hamiltonian cycle through a sparse graph of $n$ points to determine the shift register path. The original LUT-OPT paper correctly notes that this is possible, but even for reasonable values of $n$ it can become computationally infeasible. In defining the LUT-SR generators, the provision of a serial load chain is explicitly taken into account, by embedding a chosen cycle into the matrix $A$ from the start.

Specifically, we embed a very simple cycle of the form $i \leftarrow(i+1) \bmod r$ through the XOR bits, shown in Fig. 2. This simple cycle trivially extends through the shift registers, and allows switching between the RNG and load modes using one input bit per LUT.

Including such a simple cycle in the generators could cause statistical problems for generators when $t=3$, as there will be a simple linear dependence between adjacent output bits in cycles at a fixed lag. In an attempt to minimize this effect, an output permutation can be applied, to mix up the bits. 


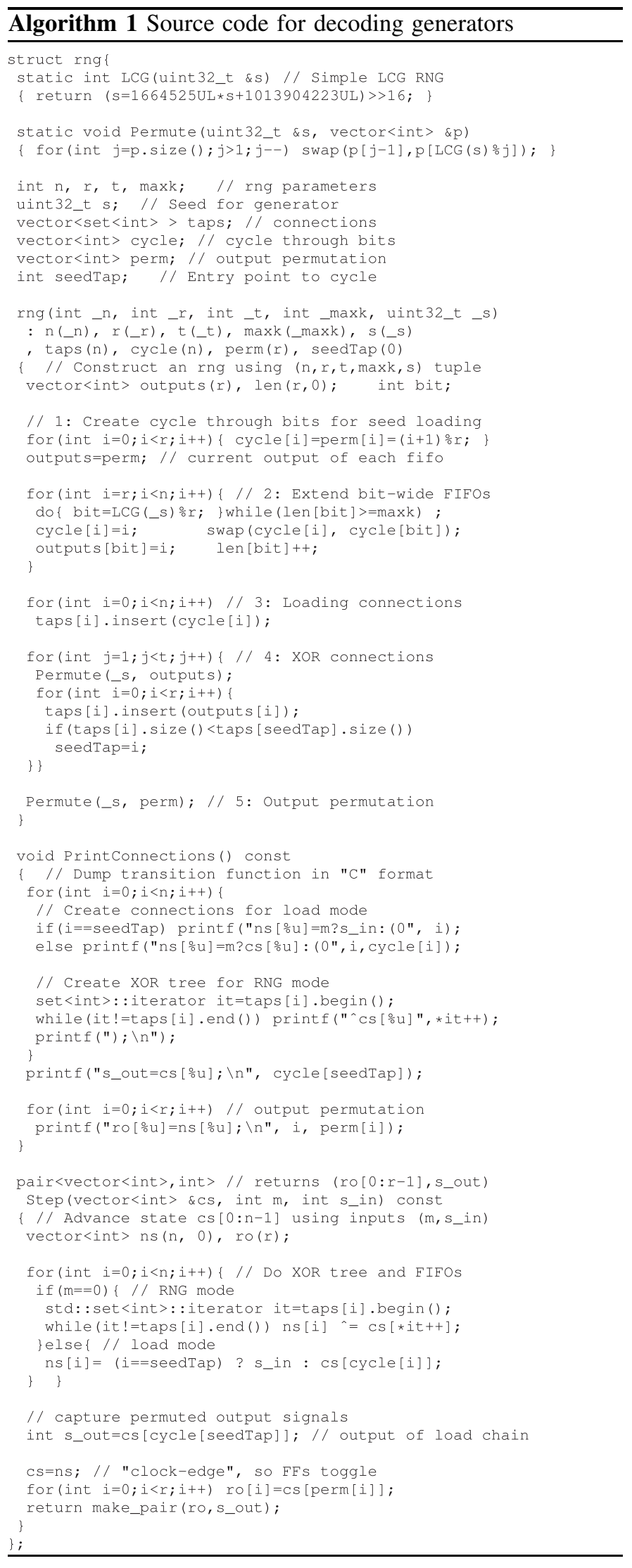

\section{Algorithm FOR DesCribing LUT-SR GENERATORS}

The broad class of LUT-SR generators as described is very general, and it is possible to construct a huge number of
// Sequential update of registers using XOR gates ns $[0]=\mathrm{m}$ ?s_in : $\left(0^{\wedge} \mathrm{Cs}\left[9{ }^{\wedge} \mathrm{Cs}[10]\right) ; / /\right.$ Load chain input $n s[1]=m ? \operatorname{cs}[6]:\left(0^{\wedge} \operatorname{cs}[6] \wedge \operatorname{cs}[11]\right)$;

$\mathrm{ns}[2]=\operatorname{m} ? \operatorname{cs}[11]:\left(0^{\wedge} \operatorname{cs}[6] \wedge \operatorname{cs}[10] \wedge \operatorname{cs}[11]\right) ;$

$\mathrm{ns}[3]=\operatorname{m?cs}[9]:\left(0^{\wedge} \operatorname{cs}[9] \wedge \operatorname{cs}[10] \wedge \operatorname{cs}[11]\right) ;$

// Sequential update of internal shift-Register bits

$\mathrm{ns}[4]=\operatorname{m} ? \operatorname{cs}[3]:\left(0^{\wedge} \operatorname{cs}[3]\right)$;

$\mathrm{ns}[5]=\operatorname{m?cs}[1]:\left(0^{\wedge} \operatorname{cs}[1]\right) ;$

$\mathrm{ns}[6]=\operatorname{m} ? \operatorname{cs}[2]:\left(0^{\wedge} \operatorname{cs}[2]\right)$;

$\mathrm{ns}[7]=\operatorname{m} ? \operatorname{cs}[0]:\left(0^{\wedge} \operatorname{cs}[0]\right)$;

$\mathrm{ns}[8]=\operatorname{m} ? \operatorname{cs}[5]:\left(0^{\wedge} \operatorname{cs}[5]\right)$;

$\mathrm{ns}[9]=\operatorname{m?cs}[7]:\left(0^{\wedge} \operatorname{cs}[7]\right)$;

$\mathrm{ns}[10]=\operatorname{m} ? \operatorname{cs}[8]:\left(0^{\wedge} \operatorname{cs}[8]\right) ;$

ns $[11]=m ? \operatorname{cs}[4]:\left(0^{\wedge} \operatorname{cs}[4]\right)$;

// Combinatorial outputs

s_out =cs[10]; // Output of serial load chain

ro [0] =ns[3]; // Permuted output bits.

ro[1] =ns [2];

ro $[2]=n s[0]$;

ro [3] =ns [1];

Fig. 3. Pseudo-RTL output from "PrintConnections" function for tuple ( $n=$ $12, r=4, t=3, k=3$, and $s=0 \times 4 d)$.

candidate LUT-SR RNGs by randomly generating binary matrices that meet the requirements. However, this presents the problem of communicating and disseminating RNGs experienced with the LUT-OPT and LUT-FIFO papers: the matrices are far too large and complicated to be included in a publication, and so must be provided separately from this paper. Instead, the LUT-SR generator family uses a short but precise algorithm for expanding a tuple of five integers into the full RNG structure.

The algorithm takes as input a 5-tuple $(n, r, t, k$, and $s)$.

$n \quad$ Number of state bits in the RNG (period is $2^{n}-1$ ).

$r \quad$ Number of random output bits generated per cycle.

$t \quad$ XOR gate input count.

$k \quad$ Maximum shift register length.

$s \quad$ Free parameter used to select a specific generator.

The first four parameters $(n, r, t, k)$ describe the properties of the generator in terms of application requirements and architectural restrictions. The final parameter $s$ is used to select from amongst one of $2^{32}$ candidates that the algorithm can produce with the chosen values of $(n, r, t, k)$.

Please Note: arbitrary values of $s$ will not result in a valid $\mathrm{RNG}$, as the choice of $s$ is critically dependent on $(n, r, t, k)$, and modifying one or more components will break the generator. Please only use the tuples listed later in this paper.

Algorithm 1 gives the expansion algorithm and RNG as a $\mathrm{C}++$ class - the constructor takes a 5-tuple of RNG parameters and expands them into a complete description of the RNG, the "PrintConnections" function can then print an RTL style description of the RNG, while the "Step" function provides a software reference implementation.

The constructor expands the RNG using five stages.

1) Create Initial Seed Cycle: A cycle of length $r$ is created through the $r$ XOR gates at the output of the RNG. At this stage there are no FIFO bits, or equivalently there are $r$ FIFOs of length 0 .

2) FIFO Extension: the cycle is randomly extended until a total cycle length of $n$ is reached, by randomly selecting a FIFO and increasing its length by 1 , while maintaining the known cycle. 
3) Add Loading Connections: the known cycle is added to the graph "taps," which describes the matrix A. The cycle describes the FIFO connections completely, and also describes the first input to each of the $r$ XOR gates.

4) Add XOR Connections: the cycle provides one input for each of the XOR gates, so now the additional $t-1$ random inputs are added over $t-1$ rounds. Each round is constructed from a permutation of the FIFO outputs, which ensures that at the end each FIFO output is used at most $t$ times. Some bits will be assigned the same FIFO bit in multiple rounds, and so will have fewer than $t$ inputs: this is critical to achieve a maximum period generator, and also provides us with an entry point into the cycle for seed loading.

5) Output Permutation: the simple dependency between adjacent bits is masked using a final output permutation.

After construction, the RNG structure can be printed as RTL-style $\mathrm{C}$ using the function "PrintConnections." For example, the input tuple $(n=12, r=4, t=3, k=3$, and $s=0 \times 4 \mathrm{~d}$ ) describes a generator with period $2^{12}-1$, producing four random bits per cycle, with a maximum of three inputs per XOR gate, and a maximum FIFO depth of 3 . The reformatted and commented output is shown in Fig. 3.

Because of the very simple printing code, the format is not very pretty, but this describes a generator in terms of six variables.

ns, cs: cs is the current state of the generator, and ns is the next state of the generator. Both are $n$ bit vectors, and describe both the FF and FIFO state.

$\mathrm{m}$, s_in: These are the RNG inputs, with $\mathrm{m}$ choosing between RNG mode ( $m=0)$, and load mode $(m=1)$, s_in provides the serial load input in load mode.

ro: This is the $r$-bit random output of the generator, which is simply a permutation of the first $r$ bits of the generator state.

s_out: While loading a new state using $m=1$, this signal can be used to read the current state.

The function "Step" implements the same transition function directly in $\mathrm{C}++$, and can operate in both RNG and load mode.

The aim of this algorithm is to provide a precise specification that can be included in this paper. A more complete package of tools is available at http://www.doc.ic.ac.uk/ dt10/research/rngs-fpga-lut_sr.html. This includes functions for generating platform-independent VHDL code, which also extract the logical shifters to improve compilation performance. ${ }^{3}$ In addition, the package provides test-bench generation tools, which verify the VHDL code against the reference software, and demonstrate how to use the serial load capability.

\section{LUT-SR SEARCH PROCESS}

Algorithm 1 can expand any given tuple into a candidate generator, but only specific parameterizations produce maxi-

\footnotetext{
${ }^{3}$ Synthesis tools can extract the shifters from the output of "PrintConnections," but take a long time for large $n$.
}

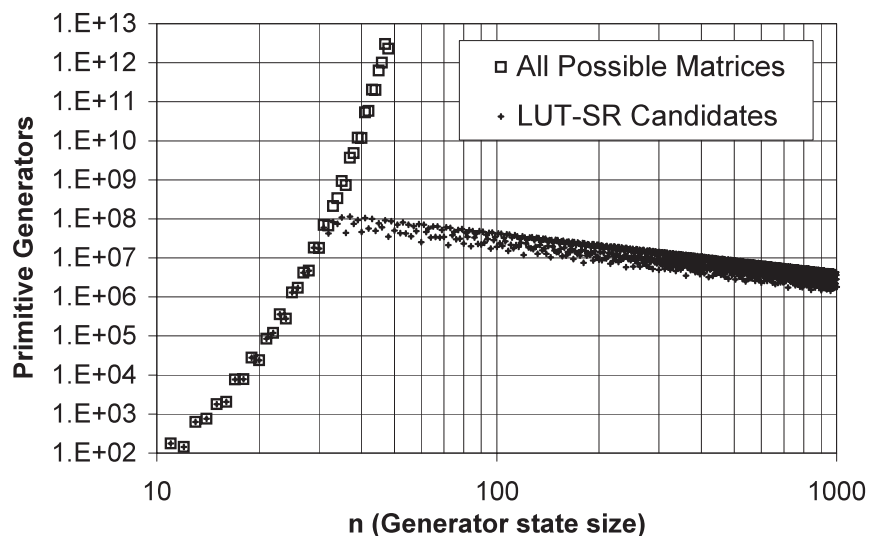

Fig. 4. Expected number of primitive generators for increased generator state size.

mum length generators, and even amongst maximum length generators some have better quality than others.

A given 4-tuple $(n, r, t, k)$ defines a set of $2^{32}$ candidate 5-tuples (as $s$ is 32-bit) which can be explored by varying $s$ exhaustively or randomly, but there is no guarantee that a valid $s$ can be found for any given 4-tuple-we might look at all $2^{32}$ tuples and find none which is maximum period. For a given $n$, there exist $\varphi\left(2^{n}-1\right) / n$ distinct degree- $n$ primitive polynomials, where $\varphi($.$) is Euler's totient function.$ Given that there are $2^{n}$ possible polynomials (including those of degree less than $n$ ), this means the probability of a random polynomial being primitive is

$$
\varphi\left(2^{n}-1\right) 2^{-n-1} .
$$

While the algorithm may generate $2^{32}$ candidates, there is no guarantee that the candidates all have distinct random polynomials. Assuming that each candidate sample represents a random sample from the set of $2^{n}$ possible polynomials, the expected number of distinct candidate polynomials is

$$
2^{n}\left(1-\left[\frac{2^{n}-1}{2^{n}}\right]^{2^{32}}\right)>0.6 \min \left(2^{n}, 2^{32}\right) .
$$

The expected primitive generators per 4-tuple is then simply the probability of any polynomial being primitive times the expected number of distinct polynomials in the 4-tuple's candidate set.

Fig. 4 shows the number of expected primitive generators for a given $n$, within both the LUT-SR candidate set and the set of all possible $n \times n$ transition matrices. For $n<32$, the number of generators is limited by the small number of possible polynomials, and many values of $s$ will lead to the same polynomial. As $n$ exceeds 32, the number of possible primitive polynomials increases greatly, but the number in the candidate set slowly decreases. ${ }^{4}$ However, the rate of decline is slow enough so that there are plenty of candidates in the set: when $n=10^{3}$, there are over a million primitive candidates, and when $n=10^{4}$, there are still hundreds of thousands.

Having established that there are plenty of primitive generators within the candidate set, the problem is now how to find

\footnotetext{
${ }^{4}$ The trend continues in the same way beyond $n=1000$, but is not shown to simplify the graph.
} 
them, that is, to implement Stage 2 of the process described in Section II-A. Practically, this means we must check whether a given concrete 5-tuple has a primitive characteristic polynomial. One approach is to build the set of feedback taps using the LUT-SR algorithm and use them to create the matrix A, from which we can then extract the characteristic polynomial. However, calculating the characteristic polynomial of an arbitrary matrix is an $O\left(n^{3}\right)$ operation, so for large $n$ this becomes impractical given the need to check thousands of random matrices.

Instead of using the matrix form, it is more efficient to extract the characteristic polynomial from the output sequence. After building the candidate generator, the generator is put into an arbitrary nonzero state and the "Step" function is used to generate a sequence of $2 n$ consecutive outputs. The Berlekamp-Massey algorithm is then used to extract the minimal polynomial from this sequence: while there are some differences between the minimal and characteristic polynomial, for the purposes of testing whether a given generator is maximum period, they can be used in the same way.

For the generator to be full period, the characteristic $P(z)$ must be a degree- $n$ primitive polynomial. Primitive polynomials must be irreducible: that is, they should not factor into smaller polynomials (a similar concept to primality for integers). If $2^{n}-1$ is a Mersenne prime, then irreducibility is sufficient to prove primitivity. However, if $2^{n}-1$ is composite, then it is also necessary to show for all prime factors $f$ of $2^{n}-1$ that $P(z)^{\left(2^{n}-1\right) / f} \neq P(z)$. This places limits on which specific values of $n$ can be chosen: if the factorization of $2^{n}-1$ is not currently known, then it cannot be used to create a maximum period generator.

The process for testing whether a given 5-tuple is a primitive generator then becomes the following:

1) expand the 5-tuple into the set of feedback taps;

2) choose a random starting state, and generate a sequence of $2 n$ successive outputs from the generator;

3) use the Berlekamp-Massey algorithm to extract the minimal polynomial $P(z)$ from the sequence;

4) if $P(z)$ has degree less than $n$, then reject;

5) if $P(z)$ is reducible, then reject;

6) for each prime factor $f$ of $2^{n}-1$, if $P(z)^{\left(2^{n}-1\right) / f}=$ $P(z)$, then reject;

7) the polynomial is primitive, so accept.

Any 5-tuple that completes this process has a known maximum period of $2^{n}-1$, so now we need to select the best from amongst this set.

\section{Quality Selection Process}

The quality criterion we use to select the best value of $s$ is based on equidistribution (see Section II-A). If a generator is $(d, l)$-distributed, then it is also $(d-1, l)$-distributed and $(d, l-1)$-distributed, where $d \geq 1$ and $l \geq 1$. The maximum dimension to which a generator is distributed to $l$ bits is $d_{l}$, with an upper bound of $d_{l}^{*}=\lfloor n / l\rfloor$. This leads to two common quality measures [3] based on the dimension gap $\delta_{l}=d_{l}^{*}-d_{l}$

$$
\Delta_{1}=\sum_{i=1}^{r} \delta_{l}, \Delta_{\infty}=\max _{i=1 . . r} \delta_{l} .
$$

When a generator has $\Delta_{1}=\Delta_{\infty}=0$, it is maximally equidistributed (ME), as it has achieved the best possible equidistribution for any generator with the same $n$ and $r$.

However, these metrics suffer from problems due to the reliance on absolute dimension differences. For example, if $n=64$, then the dimension gap $\delta_{2}=1$ means that the generator achieved a dimension of 31 rather than 32, but the same size gap at $\delta_{32}=1$ means that the generator has a dimension of 1 rather than 2. The first gap is not that significant, but the second gap means the quality has effectively halved, making it difficult to use dimension gaps to compare generators with the same $n$. Similarly, one cannot easily compare generators with different $n$ : a value of $\Delta_{\infty}=1$ is very good for a generator with $n=19937$, but is very bad for a generator with $n=32$.

The metric we used when selecting from amongst the maximum period generators found is a metric called $Q$

$$
Q_{l}=\sqrt[l]{\prod_{i=1}^{i=l} \frac{d_{l}}{d_{l}^{*}}} .
$$

This metric produces a value in the range $(0,1]$, with $Q_{r}=1$ occurring only for ME generators. It also takes into account relative changes in dimension: for $\left\{n=64, r=32, \Delta_{1}=1\right\}$, if $\delta_{2}=1$, then $Q_{32}=0.999$, while if $\delta_{32}=1$, then $Q_{32}=$ 0.978 , making it clear which one is better.

There are two main ways of calculating equidistribution: either by using lattice reductions in terms of the output sequence [7], or by using matrix operations on the state sequence [3]. The lattice methods are often faster for evaluating software generators, as they work well when $r \ll n$, and the software generators are able to generate output samples efficiently. However, generating samples from a generator optimized for hardware is relatively slow because of the complicated bitwise recurrence, while the larger $r$ values reduce efficiency.

The matrix-based methods operate by creating a matrix that relates the $d l$ bits of the $d$-tuple to the current state of the generator. The tuple we are measuring is

$$
\begin{aligned}
\mathbf{e}_{i, d, l} & =\left(\mathbf{y}_{i, 1 \ldots l}, \mathbf{y}_{i+1,1 \ldots l}, \ldots, \mathbf{y}_{i+d, 1 \ldots l}\right) \\
& =\left(\mathbf{S}_{l} \mathbf{B} \mathbf{x}_{i}, \mathbf{S}_{l} \mathbf{B A} \mathbf{x}_{i}, \mathbf{S}_{l} \mathbf{B} \mathbf{A}^{2} \mathbf{x}_{i}, \ldots, \mathbf{S}_{l} \mathbf{B} \mathbf{A}^{d-1} \mathbf{x}_{i}\right)
\end{aligned}
$$

where $S_{l}$ is an $l \times n$ matrix containing the first $l$ rows of the identity matrix. A $d l \times n$ matrix $\mathbf{E}$ can be constructed which maps the bits of $x_{i}$ to the $d l$ bits of $e_{i}$

$$
\mathbf{E}_{d, l}=\left[\begin{array}{lll}
\left(\mathbf{S}_{l} \mathbf{B}\right)^{T} & \left(\mathbf{S}_{l} \mathbf{B A}\right)^{T} \ldots\left(\mathbf{S}_{l} \mathbf{B} \mathbf{A}^{d-1}\right)^{T}
\end{array}\right]^{T} .
$$

If and only if the matrix $E_{d, l}$ has full rank, then the generator is $(d, l)$-distributed.

In principle, the matrix-based method requires $O\left(n^{4}\right)$ time, because of the need to perform $O(n)$ matrix multiplications, but in practice $\mathbf{A}$ is extremely sparse for LUT-SR generators, so the matrix-multiply cost is $O\left(n^{2}\right)$ and the actual cost of forming $E_{d, l}$ is effectively $O\left(n^{3}\right)$. The matrix rank calculation can also be performed progressively, so the echelon form of $E_{d, l}$ can be used as the starting point for echelonizing 
TABLE I

TABLES OF $s$ FOR RNGS OF FORM $(n, r, t, k=32, s)$

\begin{tabular}{|c|c||c|c|c|c|}
\hline $\mathrm{n}$ & $\mathrm{r}$ & $t=3$ & $t=4$ & $t=5$ & $t=6$ \\
\hline 1024 & 32 & $1 \mathrm{a} 5 \mathrm{eb}$ & $1562 \mathrm{~cd} 6$ & $1 \mathrm{c} 48$ & $2999 \mathrm{~b} 26$ \\
1280 & 40 & $\mathrm{c} 51 \mathrm{~b} 5$ & $4 \mathrm{ffa} 6 \mathrm{a}$ & $3453 \mathrm{f}$ & 171013 \\
1536 & 48 & 76010 & $\mathrm{c} 2 \mathrm{dc} 4 \mathrm{a}$ & $4 \mathrm{~b} 2 \mathrm{be} 0$ & $811 \mathrm{a} 15$ \\
1788 & 56 & $\mathrm{a} 2 \mathrm{aae}$ & $23 \mathrm{f} 5 \mathrm{fd}$ & $1 \mathrm{dde} 4 \mathrm{~b}$ & $129 \mathrm{~b} 8$ \\
2048 & 64 & $5 \mathrm{f} 81 \mathrm{cb}$ & 456881 & $\mathrm{bfbaac}$ & $21955 \mathrm{e}$ \\
2556 & 80 & $755 \mathrm{bac}$ & $7454 \mathrm{a} 5$ & $8 \mathrm{a} 0 \mathrm{c} 78$ & $\mathrm{cc7516}$ \\
3060 & 96 & $79 \mathrm{e} 56$ & $9 \mathrm{a} 7 \mathrm{~cd}$ & $41 \mathrm{a} 62$ & $1603 \mathrm{e}$ \\
3540 & 112 & $78 \mathrm{~d} 9 \mathrm{df}$ & $7737 \mathrm{bf}$ & 870295 & $\mathrm{~b} 850 \mathrm{c} 9$ \\
3900 & 128 & 10023 & $197 \mathrm{bf} 8$ & $\mathrm{cc} 71$ & $14959 \mathrm{e}$ \\
5064 & 160 & $42 \mathrm{f} 017$ & $3 \mathrm{~d} 31 \mathrm{e} 4$ & $43 \mathrm{c} 621$ & $51249 \mathrm{a}$ \\
5064 & 192 & $48 \mathrm{a} 92$ & $439 \mathrm{~d} 3$ & 4637 & $577 \mathrm{ce}$ \\
6120 & 224 & $3 \mathrm{e} 2834$ & $3 \mathrm{ca} 4 \mathrm{af}$ & $401 \mathrm{dfd}$ & $42 \mathrm{~d} 8 \mathrm{f} 2$ \\
8033 & 256 & $437 \mathrm{c} 26$ & 439995 & $43664 \mathrm{f}$ & $427 \mathrm{ba} 2$ \\
11213 & 384 & $\mathrm{a} 6847$ & 92228 & $\mathrm{a} 4 \mathrm{afa}$ & afd67 \\
19937 & 624 & $209 \mathrm{eb}$ & $2 \mathrm{e} 5 \mathrm{fa}$ & $2 \mathrm{fffb}$ & $25 \mathrm{c} 7 \mathrm{~d}$ \\
\hline
\end{tabular}

$E_{d+1, l}$, allowing $d_{l}$ to be iteratively determined starting from dimension 1.

The generator search and equidistribution calculations were performed using a combination of NTL [11] and M4RI [12] for dense matrix and polynomial operations, a customized version of PPSearch [13] for primitivity testing, a custom sparsematrix library optimized for RNG operations, and a custom progressive rank library for equidistribution calculations.

\section{TABLES OF LUT-SR GENERATORS}

Table I provides a list of generator tuples for a variety of useful parameterizations. To provide maximum real-world benefit, we have chosen to examine the situation, where $k=$ 32. This works well in modern FPGAs, requiring one LUT per shift register, and means that each generator needs $2 r$ LUTs and $r$ FFs. Using the shift register output directly frees up the associated FF, but reduces the clock rate slightly. For maximum speed, the final output of the shift register can be placed in an FF, increasing the resource usage to $2 r$ LUT-FFs, while allowing $600-\mathrm{MHz}+$ performance in Virtex-6 without any manual tuning.

We choose $n \sim r k$, as this means that the period increases with the number of output bits, and results in a similar equidistribution, even when large numbers of bits are generated per cycle. Note that, because we need to know the factorization of $2^{n}-1$ (see Section V), we cannot choose $n=r k$ when $r k$ becomes large, so instead choose the closest pair for which the factorization is known.

Input taps for $t=3 . .6$ are considered, as it may be preferable to use more or less taps depending on the situation. For example, a Virtex-4 generator could be implemented using $t=3$ and two SRL16s per shift register, or an RNG that does not need to be explicitly seeded could be implemented using $t=6$ in a Virtex-6. In general, $t=5$ is recommended, as this provides a high level of state mixing, while still allowing an efficient implementation in modern architectures.
TABLE II

COMPARISON OF GENERATORS BY QUALITY AND RESOURCE Us AGE

\begin{tabular}{|c|c|c|c|c|ccc|c|}
\hline Generator & $\begin{array}{c}\text { Failed } \\
\text { tests }\end{array}$ & $n$ & $r$ & $\frac{w\left(P_{z}\right)}{n}$ & RAM & LUT & FF & $\frac{r}{\text { LUT }}$ \\
\hline TAUS-113 [3] & 6 & 113 & 32 & 0.43 & 0 & 87 & 208 & 0.37 \\
TT-800 [14] & 14 & 800 & 32 & 0.33 & 2 & 162 & 162 & 0.26 \\
MT-19937 [15] & 2 & 19937 & 32 & 0.01 & 2 & 278 & - & 0.12 \\
WELL-19937 [16] & 2 & 19937 & 32 & 0.43 & 4 & 633 & 537 & 0.05 \\
LFSR-160 [1] & 13 & 160 & 32 & 0.03 & 0 & 448 & 384 & 0.07 \\
LUT-OPT [1] & 4 & 1024 & 1024 & 0.23 & 0 & 1024 & 1024 & 1.00 \\
LUT-FIFO [2] & 2 & 11213 & 521 & 0.50 & 1 & 539 & 611 & 0.97 \\
\hline LUT-SR $(t=5)$ & 4 & 1024 & 32 & 0.45 & 0 & 64 & 64 & 0.50 \\
LUT-SR $(t=5)$ & 2 & 19937 & 624 & 0.50 & 0 & 1248 & 1248 & 0.50 \\
\hline
\end{tabular}

The generators listed in the table have been tested using the Crush and BigCrush empirical test batteries from the TestU01 package [9]. These batteries perform extremely stringent statistical tests, and the LUT-SR generators pass all of them convincingly, except the matrix rank and linear complexity tests. This is a known problem with all binary linear generators, including the Mersenne Twister, WELL, LFSRs, and Combined Tausworthe generators, all of which will fail such tests. However, in practice these specific flaws rarely affect simulations: the Mersenne Twister has been used in thousands of applications without problems, so the same should be true of the LUT-SR generators.

\section{EVALUATION OF LUT-SR GENERATORS}

Table II provides a comparison of a number of RNGs suggested for FPGAs. The TAUS-113 [3], TT-800 [14], MT-19937 (Mersenne Twister) [15], and WELL-19937 [16] generators are all software generators ported to hardware, while the LUTOPT [1], LUT-FIFO [2], and LUT-SR are all designed specifically for FPGAs. The LFSR-160 uses 32-bit-wide LFSRs in parallel, which has an efficient implementation in both hardware and software.

The table includes three quality metrics: "Failed Tests" is the number of tests from the BigCrush battery that are failed, " $n$ " gives the period of the generators, and $w\left(P_{z}\right) / n$ is the ratio of $1 \mathrm{~s}$ to $0 \mathrm{~s}$ in the characteristic polynomial. All else being equal, larger $n$ implies higher quality, while $w\left(P_{z}\right) / n$ should be relatively close to 0.5 , but these are only relative metrics that are difficult to interpret on an absolute scale.

The number of tests failed in BigCrush is a more useful absolute metric of quality, as the tests detect specific quality problems in the generator. TT- 800 and LFSR-160 do particularly badly, failing multiple tests, including those that do not depend on the linear structure of the generator: that is, nonrandomness beyond that required by the generation method. All the other methods only fail the linear-complexity and matrix-rank tests, with the difference in numbers of failures due to the differences in periods. BigCrush uses a number of different parameterizations of the tests, and longer period generators are able to pass tests that look for predictable linear complexity in smaller sub-sequences. 


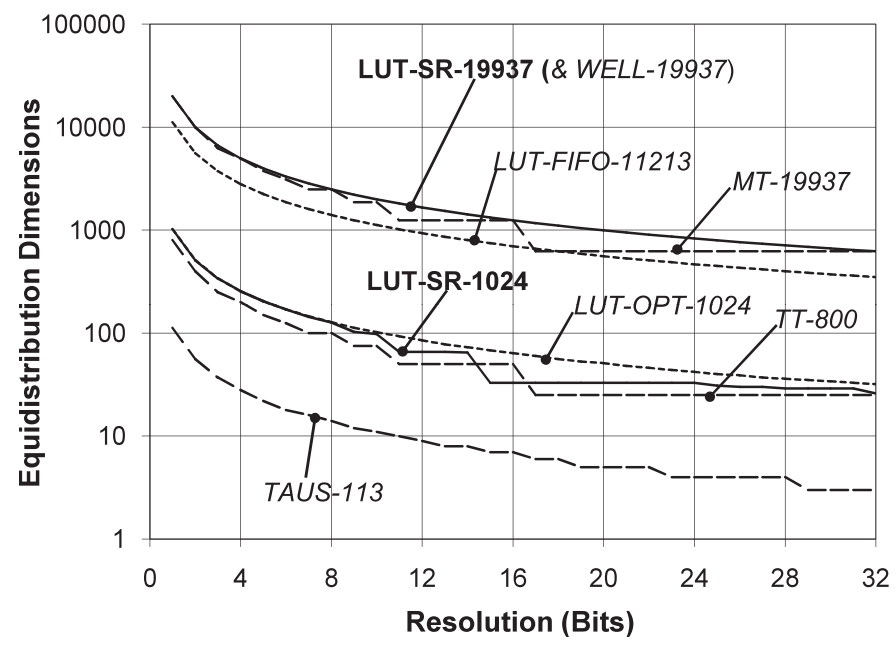

Fig. 5. Number of equidistributed dimensions (quality) for increasing resolution (number of MSBs).

Fig. 5 compares the equidistribution of the different generators over the first 32 bits. Looking at the long period generators, WELL-19937 and LUT-SR-19937 have effectively the same equidistribution, although LUT-SR-19937 does not quite achieve the ME property-it achieves $\Delta_{\infty}=1$ and $\Delta_{1}=3$ over the first 32 MSBs. At greater resolutions, $\delta_{4}=\delta_{8}=\delta_{15}=\delta_{66}=\delta_{89}=1$, and for the first 128 bits we have $\Delta_{\infty}=1$ and $\Delta_{1}=5$. This equidistribution is considerably better than MT-19937, which develops large enough dimension gaps at 16 bits to drop to the quality of the much lower period LUT-FIFO-11213.

In the lower period group, the LUT-OPT-1024 generator does not quite achieve the ME property but still has $\Delta_{\infty}=1$ over the first 32 bits, while the LUT-SR-1024 generator does not do as well. For the first 10 bits it is ME, but then develops a small dimension gap at 10 bits followed by a steeper drop at 15 bits. However, it is distributed to 22 dimensions at 32-bit resolution, which is an order of magnitude more than TAUS-113.

The FPGA-optimized generators all provide the best performance in terms of quality versus resources: amongst the lower period generators, the LUT-OPT generator uses the absolute minimum resources of one LUT per generated bit, but unless one wishes to use all 1024 generated bits it is far less efficient than the LUT-SR-1024, the LUT-FIFO generator can provide very long periods to match those of the Mersenne Twister, but requires the use of a block RAM, and now the new LUT-SR generator provides a useful mid-point between the two, with a good balance between resource utilization and good quality.

The software RNGs provide the best equidistribution, but it is difficult to justify the small improvement in quality over the LUT-SR RNGs given the cost in resources; the WELL-19937 generator requires four block RAMs to remove a dimension gap of $\Delta_{1}=3$, and only achieves $1 / 19$ th of the throughput. The ability to customize the number of generated bits is also a particular advantage in FPGA applications, as it is rare that precisely 32 bits are needed.

In terms of performance, all the FPGA-optimized generators are also intrinsically fast: both the LUT-OPT and LUT-SR generators have a LUT-FF-LUT critical path, and provide post-place-and-route clock rates of $600 \mathrm{MHz}+$ in Virtex-6 without any optimization, even for large values of $r$. The LUT-FIFO generators are typically limited by the clock rate of the block-RAM providing the FIFOs, and so can achieve $550 \mathrm{MHz}+$.

\section{CONCLUSION}

This paper presented a family of FPGA-optimized uniform RNGs, called LUT-SR RNGs. These RNGs take advantage of the ability to configure LUTs as independent shift registers, allowing high-quality long-period generators to be implemented using only a small amount of logic. In addition, the period and quality scale with the number of output bits, unlike generators adapted from software.

A key advantage of the LUT-SR generators over previous FPGA-optimized uniform RNGs is that they can be reconstructed using a simple algorithm, which is contained in this paper. In concert with the tables of maximum period generators, this allows FPGA engineers to use the new RNGs without needing to find generator instances themselves.

\section{REFERENCES}

[1] D. B. Thomas and W. Luk, "High quality uniform random number generation using LUT optimised state-transition matrices," J. VLSI Signal Process., vol. 47, no. 1, pp. 77-92, 2007.

[2] D. B. Thomas and W. Luk, "FPGA-optimised high-quality uniform random number generators," in Proc. Field Program. Logic Appl. Int. Conf., 2008, pp. 235-244.

[3] P. L'Ecuyer, "Tables of maximally equidistributed combined LFSR generators," Math. Comput., vol. 68, no. 225, pp. 261-269, 1999.

[4] D. B. Thomas and W. Luk, "FPGA-optimised uniform random number generators using luts and shift registers," in Proc. Int. Conf. Field Program. Logic Appl., 2010, pp. 77-82.

[5] M. Matsumoto and T. Nishimura, "Mersenne twister: A 623 dimensionally equidistributed uniform pseudo-random number generator," ACM Trans. Modeling Comput. Simulat., vol. 8, no. 1, pp. 3-30, Jan. 1998.

[6] M. Saito and M. Matsumoto, "SIMD-oriented fast mersenne twister: A 128-bit pseudorandom number generator," in Monte-Carlo and QuasiMonte Carlo Methods. New York: Springer-Verlag, 2006, pp. 607-622.

[7] F. Panneton, P. L'Ecuyer, and M. Matsumoto, "Improved long-period generators based on linear recurrences modulo 2," ACM Trans. Math. Software, vol. 32, no. 1, pp. 1-16, 2006.

[8] M. Matsumoto and Y. Kurita, "Twisted GFSR generators II," ACM Trans. Modeling Comput. Simulat., vol. 4, no. 3, pp. 254-266, 1994.

[9] P. L'Ecuyer and R. Simard. (2007). TestU01 Random Number Test Suite [Online]. Available: http://www.iro.umontreal.ca/ imardr/indexe.html

[10] F. Panneton, P. L'Ecuyer, and M. Matsumoto, "Improved long-period generators based on linear recurrences modulo 2," ACM Trans. Math. Software, vol. 32, no. 1, pp. 1-16, 2006.

[11] V. Shoup. (1997, Jan. 15). NTL: A Library for Doing Number Theory [Online]. Available: http://www.shoup.net/ntl/

[12] M. Albrecht and G. Bard. (2010). The M4RI Library - Version 20100817 [Online]. Available: http://m4ri.sagemath.org

[13] S. Duplichan. (2003). PPSearch: A Primitive Polynomial Search Program [Online]. Available: http://users2.ev1.net/ sduplichan/ primitivepolynomials/

[14] V. Sriram and D. Kearney, "A high throughput area time efficient pseudo uniform random number generator based on the TT800 algorithm," in Proc. Int. Conf. Field Program. Logic Appl., 2007, pp. 529-532.

[15] S. Konuma and S. Ichikawa, "Design and evaluation of hardware pseudorandom number generator mt19937," IEICE Trans. Inf. Syst., vol. 88, no. 12, pp. 2876-2879, 2005.

[16] Y. Li, P. C. J. Jiang, and M. Zhang, "Software/hardware framework for generating parallel long-period random numbers using the well method," in Proc. Int. Conf. Field Program. Logic Appl., Sep. 2011, pp. 110-115. 
David B. Thomas (M'06) received the M.Eng. and Ph.D. degrees in computer science from Imperial College London, London, U.K., in 2001 and 2006, respectively.

$\mathrm{He}$ has been a Lecturer with the Electrical and Electronic Engineering Department, Imperial College London, since 2010. His current research interests include hardware-accelerated cluster computing, field-programmable gate array-based Monte Carlo simulation, algorithms and architectures for random number generation, and financial computing.
Wayne Luk (F'09) received the M.A., M.Sc., and D.Phil. degrees in engineering and computing science from the University of Oxford, Oxford, U.K.

$\mathrm{He}$ is a Professor of computer engineering with Imperial College London, London, U.K., and a Visiting Professor with Stanford University, Stanford, CA, and Queens University Belfast, Belfast, U.K. His current research interests include theory and practice of customizing hardware and software for specific application domains, such as multimedia, networking, and finance. 